\title{
Numerical Study of the Effect of the Penetration of a Crack in the Matrix of a Composite
}

\author{
Taieb Nehari \\ Dept. of Mech. Eng. \\ Djillali Liabes University of \\ Sidi Bel Abbes, Algeria \\ nehari1976@gmail.com
}

\author{
Abdelkader Ziadi \\ Lab. of Smart. St. \\ Cent. Univ. Ain \\ Temouchent, Algeria \\ aekziadi@yahoo.com
}

\author{
Djamel Ouinas \\ Dept. of Mech. Eng. \\ University of Mostaganem, \\ Algeria \\ douinas@netcourrier.com
}

\author{
Benali Boutabout \\ Dept. of Mech. Eng. \\ Djillali Liabes University of \\ Sidi Bel Abbes, Algeria \\ bboutabout@yahoo.fr
}

\begin{abstract}
In this numerical investigation, the effect of the penetration of a crack in a matrix reinforced by aluminum silicon carbide particles in a composite is studied in order to determine the thermo-mechanical behavior under the effect of different temperature gradients during cooling. To realize this, the thermal residual stresses are calculated by considering a wide range of cracks of different penetrations. The results of this investigation compared to a case without geometric discontinuity, have revealed no meaningful effect of the distribution of the stresses along a main direction perpendicular to the direction of the crack. On the other hand, regarding the distribution of the stresses along the plane of the crack and in vicinity of the particle, results show that the penetration of the crack in the matrix causes an asymmetry.
\end{abstract}

Keywords- metal matrix composites; Al/SiC; thermal residual stress; penetration; crack

\section{INTRODUCTION}

Since the last decade, metal matrix composite (MMC) materials have become increasingly important in enormous applications in the aerospace, automotive and military industries, due to their high strength-to-density ratio and excellent wear resistances $[1,2]$. The main concern about MMCs is related to relatively high states of residual stresses. During cooling down from the manufacturing temperature to room temperature, due to the fact that the constituent materials have different coefficients of thermal expansion, thermal residual stresses are produced, and this affects the mechanical behavior of MMCs [3].

Several analyses of residual stresses have been carried out, both experimentally using neutron diffraction and X-ray diffraction and theoretically, by computer simulation [4]. Nowadays, there is a wide variety of modeling approaches to the behavior of MMC. Most of them are based on the distinct properties of the constituent materials - the matrix and the reinforcement materials. These models are micromechanical models and are based on the topology and geometrical distribution of the reinforcement components.

Moreover, the micromechanical analysis provides better understanding of formation and distribution of thermal residual stress within the MMCs. The distribution of these stresses in the MMCs depends on several parameters such as inclusion shape, particles volume fraction, cooling rate during manufacturing process, and fabrication temperature.

A spherical symmetric model has been employed to calculate thermal residual stresses in $\mathrm{Al} / \mathrm{SiC}$ particle metal matrix composites [4]. The numerical analysis revealed that residual stresses within the matrix increases when the particulate volume fraction is enhanced. Meijer et al. [5] have studied the effects of inclusion shape on residual stresses of MMCs by finite element analysis. Results showed that the use of a cube shaped particle, with sharp corners and edges in the unit cell model, lead to much greater initial hardening behavior than the spherical inclusions and therefore to a greater $0.2 \%$ offset yield stress due to stress/strain localization at the particle corners and edges.

The elastic behavior of materials is significantly affected by the presence of defects which can entail the weakening of the structure and cause its destruction. Strong stress concentrations arising from geometrical or metallurgical failings, may result in the appearance of microcracks. Indeed, fracture is the consequence of the various mechanisms related to the development of the damaged zones [6]. The presence of inclusions is also investigated, as they are responsible for stress concentration, crack initiation and propagation, and therefore for the fracture of the material [7]. Experimental observations on metal-matrix composites reinforced with particles have demonstrated that damage is always generated at the reinforcement space $[8,9]$.

According to [10], further improvements in the modeling of particle-reinforced composites should aim at including the damage effect, which is known to control many critical mechanical properties such as ductility and fracture toughness. From the above literature review and to the best of our knowledge the effect of the penetration of a crack on the thermo-mechanical behavior of a metal matrix composite is not clear.

The present paper deals with the investigation and the analysis of the effect of the penetration of a micro-crack on the thermo-mechanical behavior of a microstructure of a composite of aluminum matrix reinforced with silicon carbide particles subjected to different temperature gradients (cooling process). This was done using the finite element method to numerically 
predict the residual thermal stresses acting on the microstructure. Only the damage in the aluminum matrix was considered in the present investigation. The simulations were done with Abaqus [11].

\section{MODELLING PROCEDURE}

Until now, it is still not practical to model a three dimensional random distribution of reinforcement particles, of various geometries in a matrix. Therefore, to overcome this difficulty, the unit cell analysis method is used. In this study we use a special design of matrix (Al) and reinforced particles ( $\mathrm{SiC})[12]$ as it is shown in Figure 1a. The length, width and thickness of our micro-structure are respectively $\mathrm{C}=160 \mu \mathrm{m}$; $\mathrm{C}=160 \mu \mathrm{m}$ and $\mathrm{C} / 2=80 \mu \mathrm{m}$. The diameter of the reinforced particle $(\mathrm{SiC})$ is equal to $\mathrm{D}=2 \times \mathrm{R}_{0}=20 \mu \mathrm{m}$. The bonding between the inclusions and the matrix is assumed to be perfect; the debonding on the inclusion-matrix interface is not studied.

The matrix (Al) was defined as an elastic-plastic material with a modulus of elasticity of $70 \mathrm{GPa}$, Poisson ratio of 0.3 , yield strength of $275 \mathrm{MPa}$ and a coefficient of thermal expansion of $23.4 \times 10^{-6} \mathrm{C}^{-1}$. The particle $(\mathrm{SiC})$ was considered as an isotropic elastic material. The particle was assumed to have a modulus of elasticity of $408 \mathrm{GPa}$ and a Poisson ratio of 0.2 , corresponding to silicon carbide $(\mathrm{SiC})$ properties. The coefficient of thermal expansion (hereafter CET) of SiC was considered as $5.12 \times 10^{-6}{ }^{\circ} \mathrm{C}^{-1}$. Therefore, the CET of aluminum is about four times higher than $\mathrm{SiC}$.

This composite has been subjected to a thermal cycle of preheating to $T_{0}$ followed by cooling to the ambient temperature. It is assumed that the absolute temperature field is homogeneous and that its evolution is given by:

$$
T(t)=T_{0}+\tilde{T} . t
$$

where $\mathrm{T}_{0}$ is the initial temperature, $t$ is time and $\widetilde{T}$ is the temperature rate, which is considered constant during cooling. Here, the initial temperature is considered for several cases $\mathrm{T}_{0}=120^{\circ} \mathrm{C}, 220^{\circ} \mathrm{C}, 320^{\circ} \mathrm{C}, 420^{\circ} \mathrm{C}, 520^{\circ} \mathrm{C}$ and $620^{\circ} \mathrm{C}$, whereas the final temperature is maintained equal to the ambient temperature $\left(\mathrm{T}_{\text {end }}=20^{\circ} \mathrm{C}\right)$, and the constant cooling rate was considered $\widetilde{T}=-100^{\circ} \mathrm{K} \cdot \mathrm{s}^{-1}$.

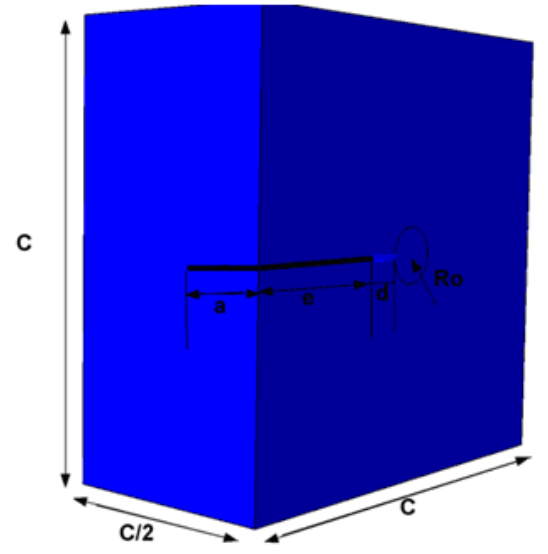

(a)

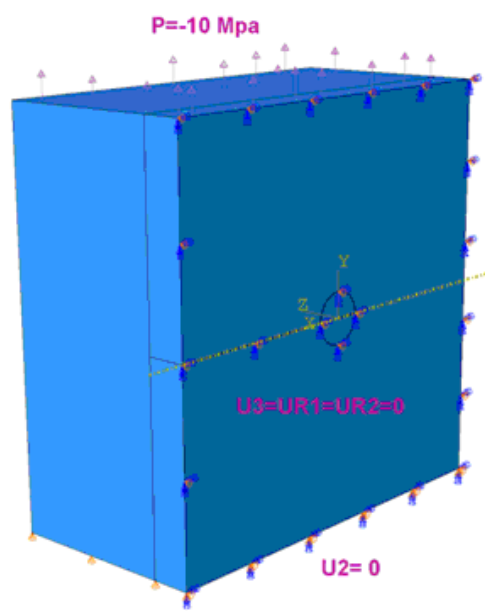

(b)

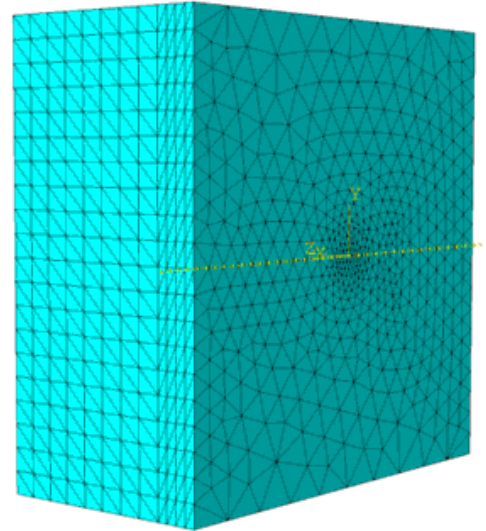

(c)

Fig. 1. Modeling a particle-reinforced composite: (a) The assumed MMC model, (b) the boundary condistions used (c) the finite element mesh

On the other hand, the boundary conditions applied to the microstructure are as follows: a fixed boundary condition of the plane surface of the bottom, a tensile pressure force of $10 \mathrm{MPa}$ is applied on the upper surface plane, and a symmetric condition has been used for the vertical plane which passes through the inclusion. However, free conditions are applied to the other boundaries planes (see Figure 1b)

The calculations were performed using ABAQUS version 6.11 [11]. Due to stress concentration, the precision of numerical method is strongly related to the quality of the designed mesh surrounding the particles and also to the zone containing the crack. Therefore, a 4 node linear tetrahedron (C3D4) finite element was used for modeling. The accuracy of the model was verified by comparing stress results with two other mesh densities, one with twice the number of elements and one with a coarse mesh having half the number of elements. The satisfactory model containing a spherical inclusion, shown in Figure 1c, consists of 52750 elements. All simulations with the meshes described above were performed with $\mathrm{Al} / \mathrm{SiC}$ composites with 20 percent volume fraction of reinforcement material.

\section{RESULTS AND DISCUSSION}

\section{A. Distribution of Residual Stress}

For the SiC/Al composite under investigation, the coefficient of thermal expansion of aluminum is about four times greater than that of SiC. Therefore, according to [12], the generations of thermal residual stresses are inevitable during cooling from the high operating temperature, resulting in 
tensile stress in the matrix and compressive ones in the reinforcement particles.

The micro-structure of the composite is subject to complex residual stresses. There are three "Principal Stresses" that can be calculated at any point, acting in the $\mathrm{x}, \mathrm{y}$, and $\mathrm{z}$ directions which are respectively $\sigma_{\mathrm{xx}}, \sigma_{\mathrm{yv}}$ and $\sigma_{\mathrm{zz}}$. The Von Mises stress, $\mathrm{VM}$, which is the combination of these three stresses into an equivalent stress, is used in this investigation. Figure 2 shows the three-dimensional distribution of the thermal residual stresses $\mathrm{V}_{\mathrm{M}}, \sigma_{\mathrm{xx}}, \sigma_{\mathrm{yy}}$ and $\sigma_{\mathrm{zz}}$ on the micro-structure of the composite for the case $a=5 \mu m, d=2 \mu m, \Delta T=300^{\circ} \mathrm{C}$.

It should be noted that the distribution of the von Mises stress is symmetrically spherical to the three planes from the center of the inclusion. It takes high values in the inclusion and at the vicinity of the matrix-particle interface. In addition, the considered stress decreases radically away from the center of the inclusion and tends to a negligible value to the extremity of the elementary volume.

On the one hand, the distribution of the $\sigma_{x x}$ stress, is symmetric in the matrix relative to the $\mathrm{x}$-axis and highly important on either side of the inclusion. This stress is negative due to the compression created on inclusion by the presence of the geometric discontinuity. On the other hand, the compressive stresses in the inclusion are twice higher than those obtained in the matrix. For this purpose, the plastic zone in the matrix surrounding the inclusion systematically generates the debonding of the interface matrix-inclusion along the $\mathrm{x}$-axis.

It should be noted that the $\sigma_{\mathrm{yy}}$ residual stresses distribution is significantly asymmetric with respect to the vertical axis in the presence of the effect of the crack appeared with the creation of the tension zone in the vicinity of the inclusion in the opposite side of crack. The compressive stresses are twice higher on both sides of the inclusion on the ordinate axis. The compressive stresses located in the particle are comparable to those shown in Figure $2 b\left(\sigma_{x x}\right)$. The difference in tensilecompressive stresses on the opposite side of the crack can produce the generation of cracks by debonding in the inclusionmatrix.

Figure $2 \mathrm{~d}$ shows the evolution of the normal stresses along the $\mathrm{z}$ axis $\left(\sigma_{\mathrm{zz}}\right)$. It is clear that the tensile stresses distribution in the matrix is homogeneous and spherical with respect to center of the inclusion whose values are comparable to the $\sigma_{\mathrm{xx}}$ stresses. The compressive stresses are in the interior of the inclusion and are of the order of five times greater than those of the tensile. Far from the particle, this stress takes low values in the matrix.

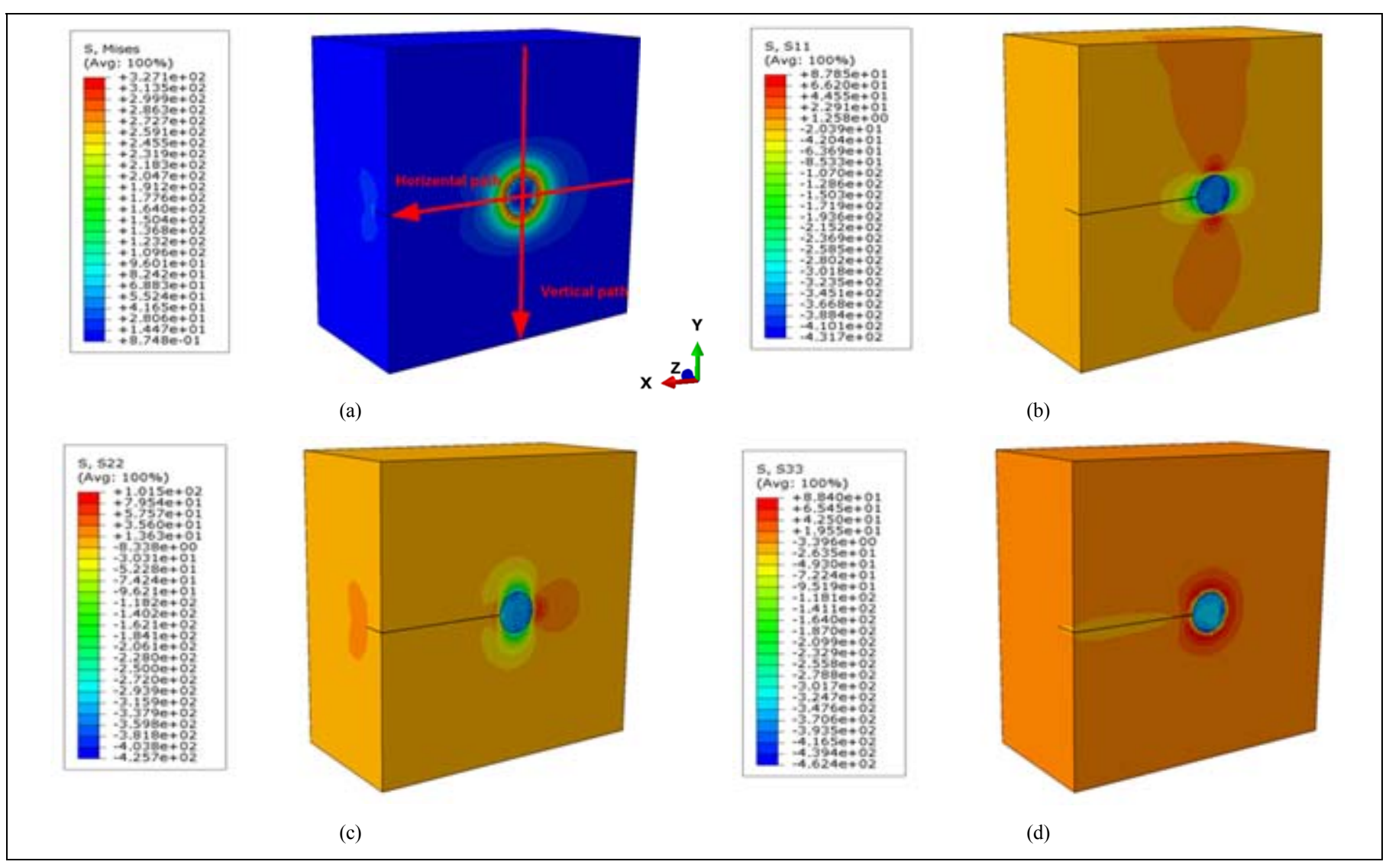

Fig. 2. Von-Mises and normal residual stress distribution for $\mathrm{d}=2 \mu \mathrm{m}, \mathrm{a}=5 \mu \mathrm{m}, \Delta \mathrm{T}=300^{\circ} \mathrm{C}$ :(a) $\sigma_{\mathrm{vm} ;}$ (b) $\sigma_{\mathrm{xx}} ;$ (c) $\sigma_{\mathrm{yy}}$ and (d) $\sigma_{\mathrm{zz}}$. 


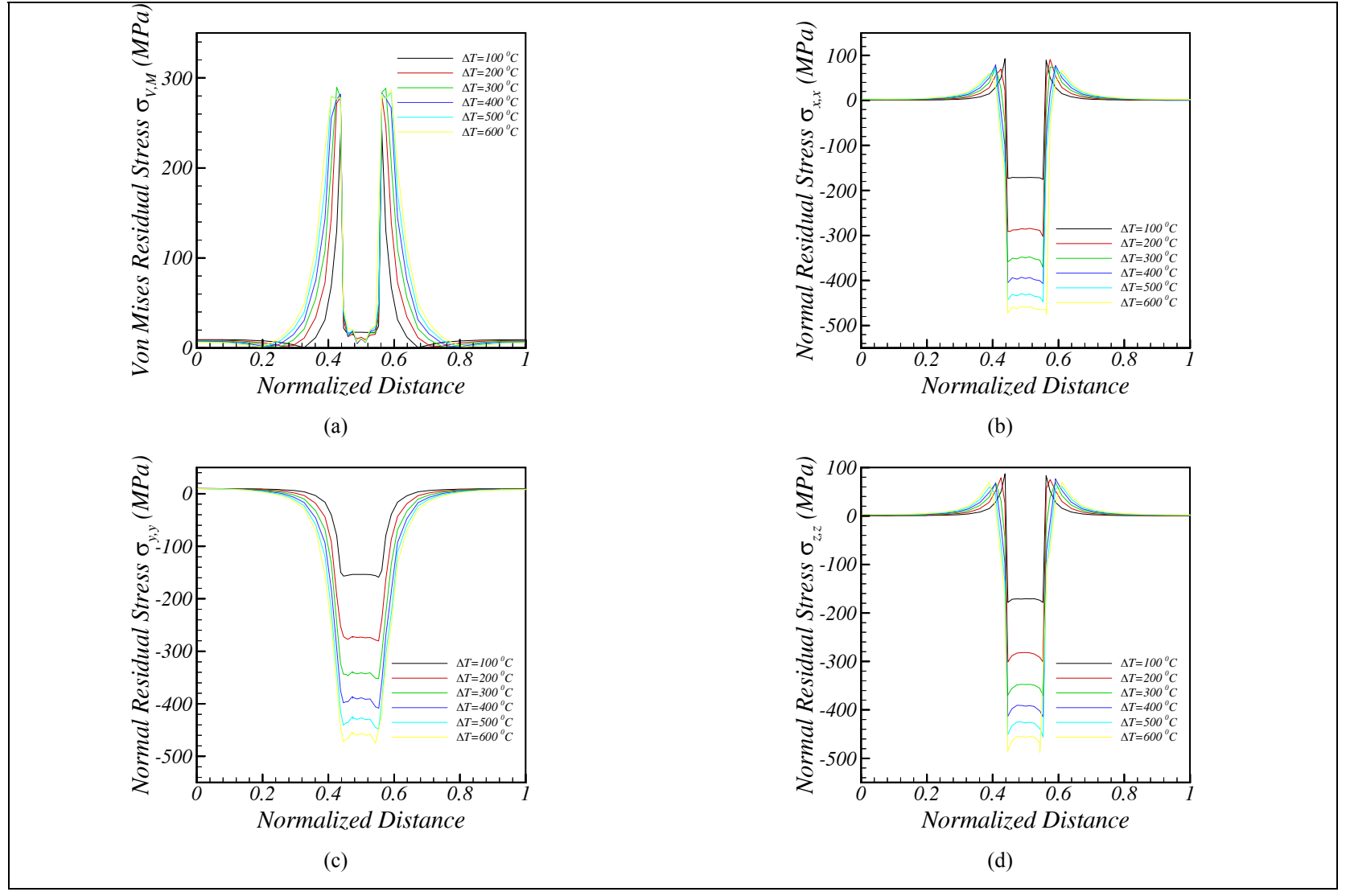

Fig. 3. Von-Mises and normal residual stress distribution along the vertical path (case without crack)

\section{B. Effect of the Temperature Gradient for the Micro- structure without Crack Case}

The composite at high temperature can plasticize, and consequently, it is necessary to model and to analyze the effect of different reheating temperatures on the generated thermal residuals stresses. First, we analyze the case without geometric discontinuity, which is displayed in Figure 3, that describes the stresses distribution along the vertical path (mentioned in Figure $2 \mathrm{a}$ ) that act on the microstructure namely VM, $\sigma_{\mathrm{xx}}, \sigma_{\mathrm{yy}}$ and $\sigma_{\mathrm{zz}}$ for different temperature gradients ranging from $100^{\circ} \mathrm{C}$ to $600{ }^{\circ} \mathrm{C}$. It should be noted that the shape of all these stresses is almost symmetrical about the center of the inclusion. The maximum stresses are proportional to the temperature gradient that replicates the difference in thermal expansion and mechanical properties of radically different materials. Thus, regardless of the different temperature gradients, the magnitudes of the stresses are insignificant away from the inclusion. It should be noted that these stresses are maximum at the interface between the inclusion and the matrix, and that they are very small or reaching zero when the distance is greater than $0.3 \mu \mathrm{m}$. It is clear that the temperature gradient has a direct and significant effect on the stresses in the vicinity close to the inclusion.

The Von Mises stresses decrease significantly within the particle to a value close to zero at the center of the particle which shows that the effect of the temperature gradient is negligible within the inclusion. An inverse behavior occurs for the $\sigma_{\mathrm{xx}}, \sigma_{\mathrm{yy}}$ and $\sigma_{\mathrm{zz}}$, stresses as they are practically constant within the particle and comparable to the maximum value obtained in the vicinity of the interface. It should be noted that the temperature gradient affects considerably on the stresses within the inclusion.

The same behavior is found for the $\mathrm{V}_{\mathrm{M}}, \sigma_{\mathrm{xx}}, \sigma_{\mathrm{yy}}$ and $\sigma_{\mathrm{zz}}$ stresses distribution along the horizontal path (as mentioned in Figure 2a) that act on the microstructure, for the same different temperature gradients as it is found for the case of vertical path (not shown here).

\section{Interaction fissure-inclusion}

The effect of crack-inclusion interaction is highlighted in the present study. It should be noted that in the case of a vertical patch, the profiles of the stresses are identical with and without the presence of geometrical discontinuity (crack). A slight difference of the stresses should be reported near the vicinity of the matrix-inclusion interface. Concerning the stresses evolution along the horizontal direction, iare found to be significantly affected by the presence of crack. The stress values are higher compared to those obtained in the no-cracks case. This means that a crack generates aditional residual stresses caused by the stresses at the crack tip and the plastic strain in the matrix in the vicinity very close to the inclusion. An increase in stresses at the surroundings of the matrix- 
inclusion interface is noticed with the presence of the crack (as shown in Figure 4). This increase is inversely proportional to the crack-inclusion gap. The simultaneous effect of the plasticity zone at the tip of the crack near the interaface and the strong stresses on the inclusion provoke an increase of the residual stresses under the effect of the temperature gradient.

Figure 5 displays the distribution of residual stresses in the vertical direction through the centre of the inclusion, in the presence of a longitudinal plane crack of width ' $\mathrm{a}=5 \mu \mathrm{m}$ '.
The gap between the tip of the crack and the particle (in the horizontal plane passing through the centre of the particle, see Figure 1a) is noted as " $d "$,. Three gap values are considered namely $d=0.2,0.5$ and $2 \mu \mathrm{m}$. It should be noted that whatever the gap " $d$ ", the paces of the stresses $\mathrm{V}_{\mathrm{M}}, \sigma_{\mathrm{xx}}, \sigma_{\mathrm{yy}}$ and $\sigma_{\mathrm{zz}}$ are almost similar under the effect of the temperature gradient with those without the presence of crack. The effect of the presence of the crack is negligible on the magnitude of stresses.
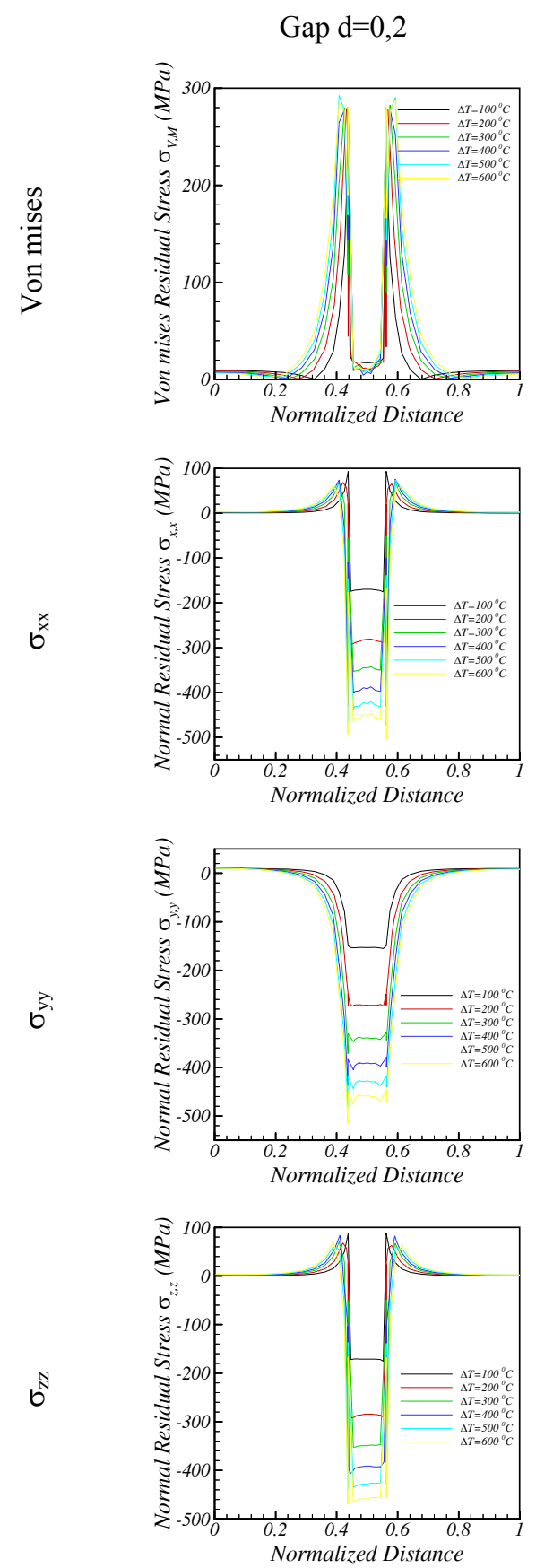

$\mathrm{d}=0,5$
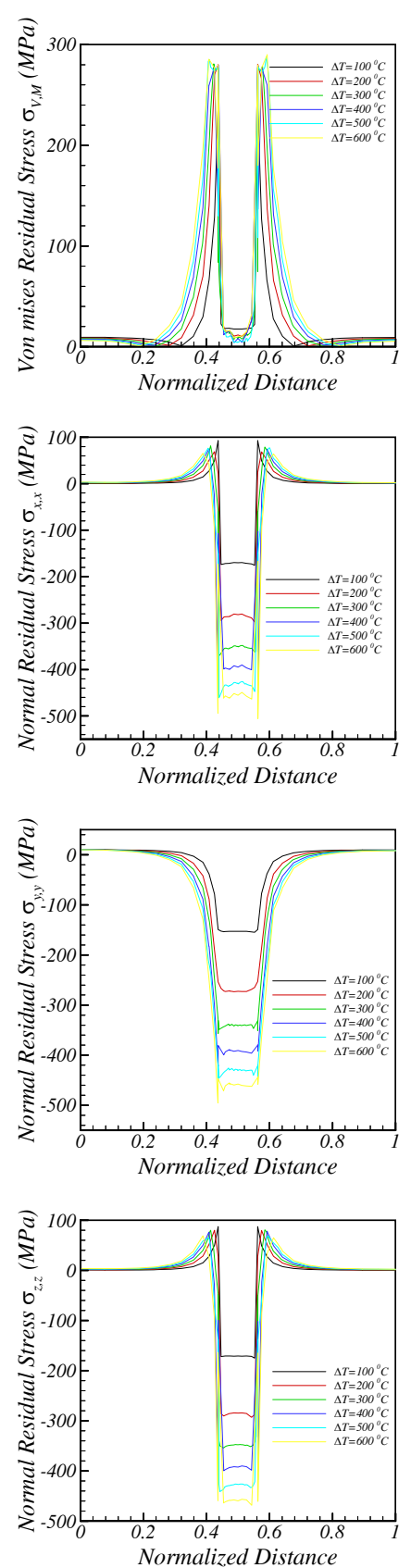

$d=2$
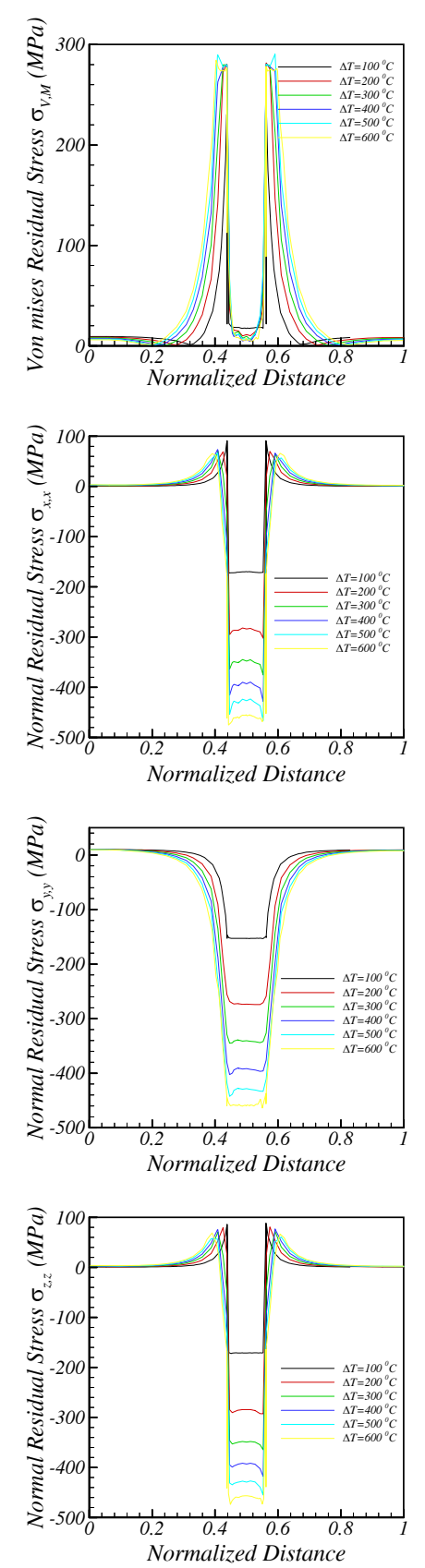

Fig. 4. Normal residual stress distribution along the vertical path in the case $\mathrm{a}=5 \mu \mathrm{m}$ and for $\mathrm{d}=0.2,0.5,2 \mu \mathrm{m}$ 


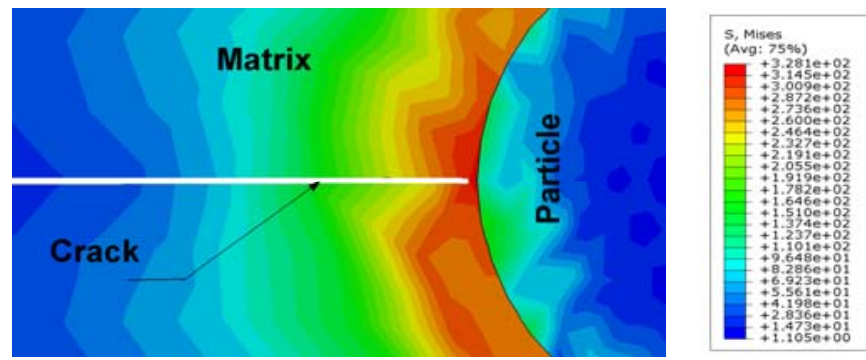

Fig. 5. Interaction Crack-particle(Crack tip region) in the case $(\mathrm{a}=5 \mu \mathrm{m}$, $\left.\mathrm{d}=0.2 \mu \mathrm{m}, \Delta \mathrm{T}=300^{\circ} \mathrm{C}\right)$
Concerning the effect the crack in the longitudinal direction, Figure 6 shows the distribution of residual stresses along such direction (path) passing through the centre of the particle, in the presence of a longitudinal plane crack of width " $a=5 \mu \mathrm{m}$ " and for different temperature gradients (from $100^{\circ} \mathrm{C}$ to $\left.600^{\circ} \mathrm{C}\right)$. As previously noted, " $d "$ is the gap of crackinclusion (see Figure 1a).

We have considered three values of the gap $(d=0.2,0.5$ and $2 \mu \mathrm{m})$. For the three cases of Figure 6, we see that the four stresses $\mathrm{V}_{\mathrm{M}}, \sigma_{\mathrm{xx}}, \sigma_{\mathrm{yy}}, \sigma_{\mathrm{zz}}$ have the same shape, and variation under the effect of temperature gradient, but are invariable in
Gap $d=0,2$

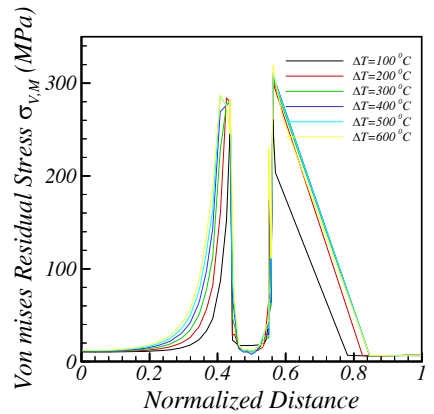

$0^{x}$
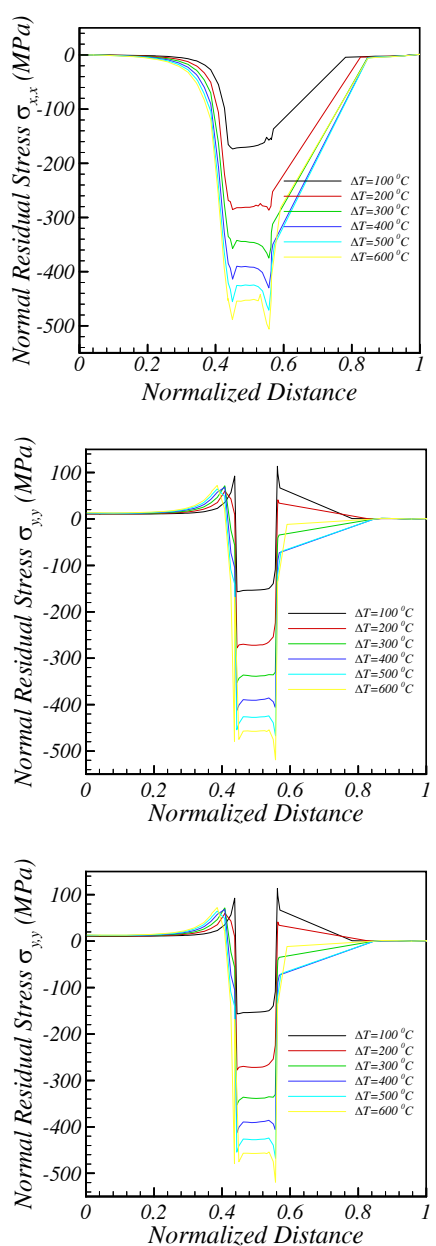

$d=0,5$
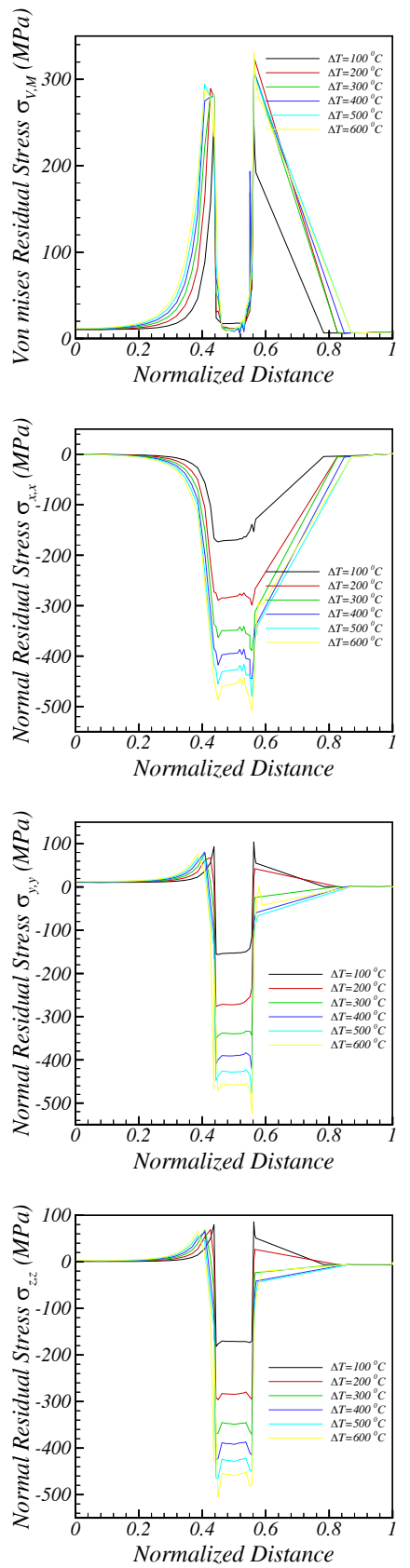

$d=2$
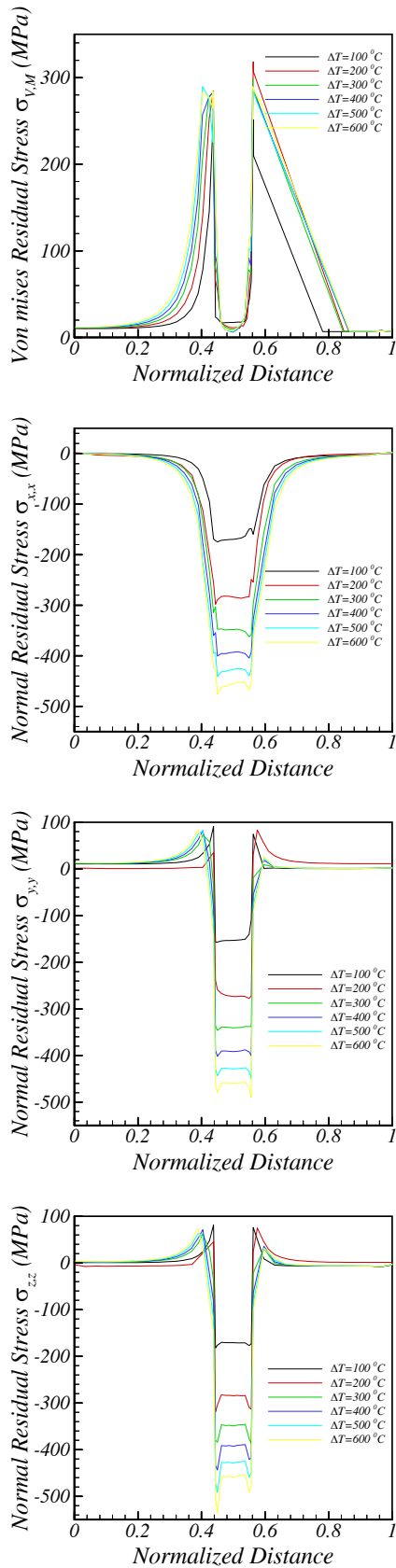

Fig. 6. Normal residual stress distribution along the longitudinal path in the case $\mathrm{a}=5 \mu \mathrm{m}$ and for $\mathrm{d}=0.2,0.5,2 \mu \mathrm{m}$ 
comparison with the no-cracks case. In contrast, in the vicinity close to the particle, we notice that the penetration of the crack in the matrix causes an asymmetry of the stresses distribution due to the presence of the geometric discontinuity (crack). It is noted that the effect of the gap is more dominant for small and medium values and thus, a more significant asymmetry in the distribution of stresses $V_{M}$ and $\sigma_{x x}$. Concerning the extreme values of stresses, it is noted that the reduction of the gap affects only the stress $\sigma_{\mathrm{zz}}$ which it falls slightly with the reduction of the " $d$ " gap (or the increase penetration crack)

\section{CONCLUSION}

The objective of this investigation is to determine the influence of crack penetration and gradient temperature on the generation of residual thermal stresses and the subsequent mechanical behaviour for the $\mathrm{Al}-\mathrm{SiC}$ composite. The present stuy is purely numeric and uses a three dimensional finite element method. The following conclusions are drawn from the results presented above:

- The thermal residual stresses have high values in the inclusion and in close vicinity to the particle-matrix interface and decrease gradually as we move away from the centre of the inclusion until they vanish to the limit of the microstructure.

- For the distribution of the stresses VM, $\sigma_{\mathrm{xx}}, \sigma_{\mathrm{yy}}, \sigma_{\mathrm{zz}}$ along the vertical path, it is noted that whatever the gap " $d$ ", the profiles of the stresses are almost identical under the effect of temperature gradient with those found in a no-cracks case, and also the temperature gradient increases significantly the intensity of stresses specially for $\sigma_{\mathrm{xx}}, \sigma_{\mathrm{yy}}$, $\sigma_{\mathrm{zz}}$.

- For the distribution of the four stresses (VM, $\left.\sigma_{\mathrm{xx}}, \sigma_{\mathrm{yy}}, \sigma_{\mathrm{zz}}\right)$ along the longitudinal direction belonging to the plane of the crack, we notice that they have the same shape and variation under the effect of the temperature gradient and similar to those found for the no-cracks case. On the other hand, in the vicinity of the particle we notice that the penetration of the crack in the matrix causes an asymmetry. Furthermore, the values of these stresses are significantly higher compared with those obtainedfor the no-cracks case. This means that the crack generates an additional residual stress caused by stresses at the crack tip and the plastic strain in the matrix at close vicinity of the inclusion.

\section{REFERENCES}

[1] D. J. Chellman, S. L. Langenbeck, "Aerospace applications of advanced aluminum alloys", Key Engineering Materials, Vol. 77-78, pp. 49-60, 1993

[2] M. Shanthi, C. Y. H. Lim, L. Lu, "Effects of grain size on the wear of recycled AZ91 Mg", Tribology International, Vol. 40, No. 2, pp. 335338,2007

[3] P. Kenesei, A. Borbély, H. Biermann, "Microstructure based threedimensional finite element modeling of particulate reinforced metalmatrix composites", Materials Science and Engineering A, Vol. 387389, pp. 852-856, 2004
[4] M. Surry, C. Teodosiu, L. F. Menezes, "Thermal residual stresses in particle-reinforced /viscoplastic metal matrix composites", Materials Science and Engineering A, Vol. 167, No. 1-2, pp. 97-105, 1993

[5] G. Meijer, F. Ellyin, Z. Xia, "Aspects of residual thermal stress/strain in particle reinforced metal matrix composites", Composites Part B: Engineering, Vol. 31, No. 1, pp. 29-37, 2000

[6] D. Ouinas, B. Bachir Bouiadjra, N. Benderdouche, "Interaction effect of a main crack emanating from a semicircular notch and a microcrack", Computational Materials Science, Vol. 43, No. 4, pp. 1155-1159, 2008

[7] D. Ouinas, B. Bachir Bouiadjra, N. Benderdouche, B. Ait Saadi, J. Vina, "Numerical modelling of the interaction macro-multimicrocracks in a plate under tensile stress", Journal of Computational Science, Vol. 2, No. 2, pp. 153-164, 2011

[8] J. Llorca, C. Gonzalez, "Microstructural factors controlling the strength and ductility of particle reinforced metal-matrix composites", Journal of the Mechanics and Physics of Solids, Vol. 46, No. 1, pp. 1-28, 1998

[9] J. Llorca, J. Segurado, "Three-dimensional multiparticle cell simulations of deformation and damage in sphere-reinforced composites", Materials Science and Engineering A, Vol. 365, No. 1-2, pp. 267-274, 2004

[10] J. Segurado, J. Llorca, "A new three-dimensional interface finite element to simulate fracture in composites", International Journal of Solids and Structures, Vol. 41, pp. 2977-2993, 2004

[11] ABAQUS, User's Manual, 6.11, Dassault Systèmes Simulia Corp, 2011

[12] F. Bouafia, B. Serier, B. Bachir Bouiadjra, "Finite element analysis of the thermal residual stresses of $\mathrm{SiC}$ particle reinforced aluminum composite", Computational Materials Science, Vol. 54, pp. 195-203, 2012 